\title{
Quantitative Estimates of Environmental Effects on the Star Formation Rate of Disk Galaxies in Clusters of Galaxies
}

\author{
Yutaka Fujita円 \\ Department of Physics, Tokyo Metropolitan University, \\ Minami-Ohsawa 1-1, Hachioji, Tokyo 192-03, Japan
}

\begin{abstract}
A simple model is constructed to evaluate the change of star formation rate of a disk galaxy due to environmental effects in clusters of galaxies. Three effects, (1) tidal force from the potential well of the cluster, (2) increase of external pressure when the galaxy plows into the intracluster medium, (3) high-speed encounters between galaxies, are investigated. General analysis indicates that the star formation rate increases significantly when the pressure of molecular clouds rises above $\sim 3 \times 10^{5} \mathrm{~cm}^{-3} \mathrm{~K}$ in $\sim 10^{8} \mathrm{yr}$. This is because the pressure rise makes the destruction time of the majority of molecular clouds in the galaxy less than $10^{8} \mathrm{yr}$. The tidal force from the potential well of the cluster accelerates molecular clouds in a disk galaxy infalling towards the cluster center. Thus, the kinetic pressure rises above $\sim 3 \times 10^{5} \mathrm{~cm}^{-3} \mathrm{~K}$. Before the galaxy reaches the cluster center, the star formation rate reaches a maximum. The peak is three to four times larger than the initial value. If this is the main mechanism of the Butcher-Oemler effect, blue galaxies are expected to be located within $\sim 300 \mathrm{kpc}$ from the center of the cluster. However this prediction is inconsistent with the recent observations. The increase of external pressure when the galaxy plows into the intracluster medium does not change star formation rate of a disk galaxy significantly. Thus, the increase of external pressure may not be the main mechanism of the Butcher-Oemler effect. The velocity perturbation induced by a single high-speed encounter between galaxies is too small to affect star formation rate of a disk galaxy, while successive high-speed encounters (galaxy harassment) trigger star formation activity because of the accumulation of gas in the galaxy center. Therefore, the galaxy harassment remains as the candidate for a mechanism of the Butcher-Oemler effect.
\end{abstract}

Subject headings: galaxies: clusters: general — ISM: clouds — stars: formation

\footnotetext{
${ }^{1}$ Present address; Department of Earth and Space Science, Faculty of Science, Osaka University, Machikaneyama-cho, Toyonaka, Osaka, 560-0043, Japan. fujita@vega.ess.sci.osaka-u.ac.jp
} 


\section{Introduction}

Clusters of galaxies in the redshift range of $0.2-0.5$ often exhibit an overabundance, relative to present-day clusters, of blue galaxies (Butcher \& Oemler 1978). This star formation activity is often called the Butcher-Oemler effect (BOE). Several mechanisms responsible for the effect have been suggested.

Tidal compression by the cluster potential increases the velocity dispersion of clouds in a disk galaxy (Byrd \& Valtonen 1990 ; Valluri 1993). Henriksen \& Byrd (1996) estimate the influence of tidal acceleration by the cluster potential on galaxies. They show that if the cluster potential is centrally peaked, compression of galaxy disk is significant. They find that the velocity perturbation due to the gravitational tidal forces are large enough for molecular clouds to give birth to stars.

Dressler \& Gunn (1983) suggest that a significant fraction of galaxies in a cluster undergo a rapid rise in external pressure as they plow into the hot intracluster medium. Evrard (1991) numerically confirms this possibility. He suggests that the high-pressure leads to star formation activity.

Recently, Moore et al. (1996) numerically show that successive high speed encounters, "galaxy harassment", cause a complete morphological transformation from disks to spheroidals. Since galaxy harassment is an extremely effective mechanism for fueling central region of a galaxy, it may cause star formation activity.

However, the above studies do not derive the history of star formation rate (SFR) quantitatively. In this paper, a simple method is devised to evaluate the SFR of a disk galaxy in a cluster in order to find out what mechanism drives the BOE. This model is useful to predict color and spatial distributions of the blue population in BOE clusters. The above three mechanisms, (1) tidal force from the potential well of the cluster, (2) increase of external pressure, and (3) high-speed encounters between galaxies are studied as candidates of the BOE. These mechanisms provide pressure variations of local interstellar medium (ISM) in a disk galaxy because of increase of kinetic or thermal pressure of ISM, and gas inflow towards the galaxy center. As will be shown in detail, the time scales of the variations are $\sim 10^{8}$, which reflect the dynamical time scales of a galaxy and central region of a cluster. Star formation responds this pressure variation locally. Elmegreen \& Efremov (1997) obtain a simple relation between star formation efficiency of molecular clouds of varying mass and pressure. Their results indicate that if the pressure of a cloud increases the order of a magnitude, the star formation efficiency increases and the lifetime decreases significantly. The relation does not care about the origin of the pressure. Thus, it can be applied to the above three cases (1) - (3). Since the lifetime of clouds is $\lesssim 10^{8} \mathrm{yr}$ 
(Elmegreen \& Efremov 1997), the pressure variations in the cases (1) - (3) are expected to affect the evolution of all the molecular clouds and the SFR of a disk galaxy. This will be discussed in the following sections.

The plan of this paper is as follows. In $§ I I$, a model of molecular cloud evolution is described. In $\S I I I$, the pressure evolution of a disk galaxy in a cluster is derived, and the SFR is evaluated. Finally, $\S \mathrm{IV}$ is devoted to conclusions. In that section, observational implications are commented.

\section{Molecular Cloud Models}

Molecular clouds are divided according to their initial masses, that is, $M_{\min }=M_{1}<\ldots<M_{i}<\ldots<M_{\max }$, where the lower and upper cutoffs are $M_{\text {min }}=10^{2} \mathrm{M}_{\odot}$ and $M_{\max }=10^{8.5} \mathrm{M}_{\odot}$, respectively, and their intervals are chosen that $\log \left(M_{i+1} / M_{i}\right)=0.01$. Referring to the total mass of clouds whose initial masses are between $M_{i}$ and $M_{i+1}$ as $\Delta \tilde{M}_{i}(t)$, the rate of change is

$$
\frac{d \Delta \tilde{M}_{i}(t)}{d t}=\tilde{f}_{i}\left[S_{\star}(t)+S_{\mathrm{mol}}(t)\right]-\frac{\Delta \tilde{M}_{i}(t)}{\tau\left(M_{i}, P\right)}
$$

where $\tilde{f}_{i}$ is the initial mass fraction of the molecular clouds whose initial masses are between $M_{i}$ and $M_{i+1}, S_{\star}(t)$ is the gas ejection rate from stars, $S_{\mathrm{mol}}(t)$ is the recycle rate of molecular gas, $\tau\left(M_{i}, P\right)$ is the destruction time of a molecular cloud with mass $M_{i}$ and pressure $P$, respectively.

Since the mass spectra of open clusters, globular clusters, and molecular clouds follow $N(M) \propto M^{-\alpha}$, where $\alpha=1.6-2$ (Harris \& Pudritz 1994 ; Elmegreen \& Efremov 1997), the initial mass function of molecular clouds is assumed to be $N(M) \propto M^{-2}$. Thus $\tilde{f}_{i}$ can be derived from this relation.

The gas ejection rate from stars is divided into two terms, $S_{\star}(t)=S_{s}(t)+S_{l}$. The gas ejection rate from stars with small lifetime is given by

$$
S_{s}(t)=\int_{m_{l}}^{m_{u}} \psi\left(t-t_{m}\right) R(m) \phi(m) d m,
$$

where $m$ is the stellar mass, $\psi(t)$ is the SFR, $\phi(m)$ is the IMF expressed in the form of the mass fraction, $R(m)$ is the return mass fraction, and $t_{m}$ is the lifetime of stars with mass $m$. The slope of the IMF is taken to be 1.35 (Salpeter mass function). The upper and lower mass limits, $m_{u}$ and $m_{l}$, are taken to be $50 M_{\odot}$ and $2.8 M_{\odot}$, respectively, although the lower limit of the IMF is $0.08 M_{\odot}$. The lifetime of stars and the return mass fraction are 
adopted from those in Mihara \& Takahara (1994) and Maeder (1992), respectively. Note that the lifetime of a star with $2.8 M_{\odot}$ is $4 \times 10^{8} \mathrm{yr}$, which is identical with the end time of calculations in the later sections. The gas ejection rate from stars with large lifetime, $S_{l}$, will be specified later.

The recycle rate of molecular gas is

$$
S_{\mathrm{mol}}(t)=\sum_{i}\left[1-\epsilon\left(M_{i}, P\right)\right] \frac{\Delta \tilde{M}_{i}(t)}{\tau\left(M_{i}, P\right)}
$$

where $\epsilon\left(M_{i}, P\right)$ is the star formation efficiency of a molecular cloud with mass $M_{i}$ and pressure $P$. Under simple assumptions, Elmegreen \& Efremov (1997) derive $\epsilon\left(M_{i}, P\right)$ and $\tau\left(M_{i}, P\right)$ : their results are adopted in the following. Note that the pressure $P$ includes the kinetic pressure (Elmegreen 1989 ; Elmegreen \& Efremov 1997). In our Galaxy, the kinetic pressure is larger than thermal pressure (Elmegreen 1989 ; Elmegreen \& Efremov 1997).

The SFR is described by

$$
\psi(t)=\sum_{i} \epsilon\left(M_{i}, P\right) \frac{\Delta \tilde{M}_{i}(t)}{\tau\left(M_{i}, P\right)} .
$$

Equation (11) can be solved if $P$ is given. In the next section, $P$ is determined for several cases. Then the SFRs are calculated form Equations (四) - (四).

\section{Evolution of SFR of Disk Galaxies in a Clusters of Galaxies}

\subsection{General Analysis}

In this subsection, the evolution of pressure is treated as a parameter to find the relation between the evolution of pressure and the SFR of a disk galaxy.

Three cases are considered, that is, the final value of $P$ is $P_{f}=10,100$, and $1000 \mathrm{P}_{\odot}$ (Figure 1(a)). The pressure is given in units of $\mathrm{P}_{\odot}=3 \times 10^{4} \mathrm{~cm}^{-3} \mathrm{~K}$ which is the total pressure in the solar neighborhood (Elmegreen \& Efremov 1997). The pressure $P$ increases for $t>0$. For $t<0$, the model galaxy is assumed to be at $P=1 \mathrm{P} \odot$

The calculation starts at $t=-10^{9} \mathrm{yr}$. At that time, the total mass of molecular clouds in the model galaxy, $M_{\text {tot }}$, is $10^{9.3} \mathrm{M}_{\odot}$, and the mass spectrum of molecular clouds is $\propto M^{-2}$. For $t<0$, ignoring Equation (2), the source term $S_{\star}$ in Equation (1) is artificially fixed at $S_{0}=6 \mathrm{M}_{\odot} \mathrm{yr}^{-1}$ which is the SFR of our Galaxy (Gústen \& Mezger 1983). After the calculation starts, the mass spectrum is modified because the destruction time of 
molecular clouds depends on their mass (Elmegreen \& Efremov 1997). Until $t=0$, the destruction and formation rates of molecular clouds become identical to within $3 \%$. At $t=0, M_{\text {tot }}=2.4 \times 10^{9} \mathrm{M}_{\odot}$, and the mass spectrum of molecular clouds is $\propto M^{-1.7}$. The total mass of molecular clouds is similar to that of our Galaxy $\left(\sim 2 \times 10^{9} \mathrm{M}_{\odot}\right.$, Larson 1987). Note that for $t>0$ the SFR history normalized by $S_{0}$ is independent of the value of $S_{0}$. The gas ejection rate from stars with large lifetime is given by $S_{l}=S_{0}-S_{s}(0)$ for $t>0$ and for fixed $P_{0}$.

The evolution of pressure, SFR, and the total mass of molecular clouds are indicated in Figure 1. As is shown, the SFRs and the time at which it become maximum depends on $P(t)$. Taking the case where $P_{f}=10 \mathrm{P}_{\odot}$ as an example, the SFR can be explained as follows. The pressure $P$ reaches $10 \mathrm{P}_{\odot}$ at $t=10^{8} \mathrm{yr}$. When $P=10 \mathrm{P}_{\odot}$, the mass of a molecular cloud whose destruction time is less than $10^{8} \mathrm{yr}$ is $\lesssim 10^{8} \mathrm{M}_{\odot}$ (see Figure 4 in Elmegreen \& Efremov 1997). On the other hand, when $t=0$, the total mass of molecular clouds whose individual mass is less than $10^{8} \mathrm{M}_{\odot}$ is $1.6 \times 10^{9} \mathrm{M}_{\odot}$. The ratio, $1.6 \times 10^{9} \mathrm{M}_{\odot} / 10^{8} \mathrm{yr}=16 \mathrm{M}_{\odot} \mathrm{yr}^{-1}$, nearly corresponds to the peak of the SFR (Figure $1(\mathrm{~b}))$. In the case where $P_{f}=100 \mathrm{P} \odot$, the SFR reaches a maximum before $P$ becomes $100 \mathrm{P}_{\odot}\left(t=2 \times 10^{8} \mathrm{yr}\right)$. This is because all molecular clouds $\left(\leq 10^{8.5} \mathrm{M}_{\odot}\right)$ are affected by the pressure increase before $t=2 \times 10^{8} \mathrm{yr}$, since the destruction time of a molecular cloud with $M=10^{8.5} \mathrm{M}_{\odot}$ is $\sim 10^{8}$ yr when $P=100 \mathrm{P}_{\odot}$. In fact, $M_{\text {tot }}$ is significantly reduced when $t=2 \times 10^{8}$ (Figure $1(\mathrm{c})$ ). Therefore, the peak of the SFR is determined by the balance between the time scale of pressure increase and the destruction time of clouds. Moreover, Figure 1(b) shows that the evolution of SFR is not very sensitive to $P_{f}$, if it is larger than $100 \mathrm{P} \odot$ and if the time scale of pressure increase is $\sim 10^{8} \mathrm{yr}$. This is because almost all of the clouds are destroyed regardless of $P_{f}$.

In summary, when pressure increases on a time scale of $10^{8} \mathrm{yr}$, the $\mathrm{SFR}$ rises significantly if $P_{f} \gtrsim 10 \mathrm{P}_{\odot}$ but reaches the ceiling at $P_{f} \sim 100 \mathrm{P} \odot$. The above arguments should be true for all of the BOE mechanisms treated in the following sections, because this model does not care about the origin of pressure.

\subsection{A Radially Infalling Galaxy}

A radially infalling disk galaxy is affected by tidal force from the cluster potential well. Molecular clouds in the galaxy are accelerated. Thus the pressure $P$ from random cloud motions increases. 
Three types of mass profiles in the central region of the cluster are investigated;

$$
\begin{gathered}
M_{\mathrm{cl}}(<r)=1.2 \times 10^{14} \mathrm{M}_{\odot}\left[\ln \left[\frac{r}{r_{\mathrm{GL}}}+\left(1+\frac{r^{2}}{r_{\mathrm{GL}}^{2}}\right)^{1 / 2}\right]-\frac{r / r_{\mathrm{GL}}}{\left(1+r^{2} / r_{\mathrm{GL}}^{2}\right)^{1 / 2}}\right], \\
M_{\mathrm{cl}}(<r)=3.5 \times 10^{14} \mathrm{M}_{\odot}\left[\ln \left[\frac{r}{r_{\mathrm{King}}}+\left(1+\frac{r^{2}}{r_{\mathrm{King}}^{2}}\right)^{1 / 2}\right]-\frac{r / r_{\mathrm{King}}}{\left(1+r^{2} / r_{\mathrm{King}}^{2}\right)^{1 / 2}}\right], \\
M_{\mathrm{cl}}(<r)=3.0 \times 10^{15} \mathrm{M}_{\odot}\left[\ln \left(1+\frac{r}{r_{\mathrm{NFW}}}\right)-\frac{r / r_{\mathrm{NFW}}}{1+r / r_{\mathrm{NFW}}}\right] .
\end{gathered}
$$

Equations (5) is the profile inferred from gravitational lensing constraints, Equations (6) is the so-called King model profile, and Equations (7) is the profile obtained in numerical simulations done by Navarro, Frenk, \& White (1995, 1996). The characteristic radii for each are $r_{\mathrm{GL}}=50 \mathrm{kpc}, r_{\mathrm{King}}=200 \mathrm{kpc}$, and $r_{\mathrm{NFW}}=900 \mathrm{kpc}$, respectively. The first profile is the same as that in Henriksen \& Byrd (1996). The mass distributions are normalized so that the mass in side $500 \mathrm{kpc}$ is $2.5 \times 10^{14} \mathrm{M}_{\odot}$ for all distributions. When the mass profile of the cluster follows Equation (7), the profile of the intracluster gas can be fitted well by (6) except for the normalization of mass. In that case, the ratio of characteristic radii, $r_{\text {King }} / r_{\mathrm{NFW}}$, is 0.22 (Makino, Sasaki, \& Suto 1998), which is the reason for taking a radius of $900 \mathrm{kpc}$ in Equation (7).

In order to calculate the tidal acceleration and the velocity perturbation of molecular clouds, the impulse approximation is used as in Henriksen \& Byrd (1996). Strictly speaking, this approximation may not be good because the rotation period of a galaxy is comparable to the time-scale in which the galaxy crosses the central region of the cluster. However, as will be noted, the results are not very sensitive to the velocity perturbations for the models considered in this subsection.

The transverse and radial tidal accelerations are

$$
\begin{aligned}
& a_{t}=G M_{\mathrm{cl}}(<r) \frac{R}{\left[R^{2}+(r+R)^{2}\right]^{3 / 2}}, \\
& a_{r}=G M_{\mathrm{cl}}(<r)\left[\frac{1}{r^{2}}-\frac{1}{(r+R)^{2}}\right],
\end{aligned}
$$

where $G$ is the gravitational constant and $R$ is the radius of the galaxy $(=20 \mathrm{kpc})$. The velocity perturbation can be estimated as

$$
V=\int_{0}^{t} a d t
$$

where $a$ is the transverse or radial tidal acceleration. The kinetic pressure is given by $P_{\text {tidal }}=\rho_{\mathrm{d}} V^{2}$, where the average density of the galaxy disk is $\rho_{\mathrm{d}}=1.67 \times 10^{-24} \mathrm{~g} \mathrm{~cm}^{-3}$. 
The evolution of $a$ is calculated from the orbits of the galaxy in the potential well of the cluster. As an idealized case, a radially infalling galaxy is investigated. The velocity of the galaxy, $v_{\text {gal }}$, can be obtained by solving the equation of motion. At $t=0$, the position of the galaxy is $350 \mathrm{kpc}$ away from the center of the cluster. From there the galaxy radially infalls towards the center. The initial velocity is $1000 \mathrm{~km} \mathrm{~s}^{-1}$. Figure 2 shows the orbits of the galaxy.

Figures 3(a) and 4(a) show the evolution of the pressure, $P=\mathrm{P}_{\odot}+P_{\text {tidal }}$, for the three types of potentials (Equations 5, 6, and 7). The rates of pressure increase due to the radial tidal acceleration are somewhat larger than those due to the transverse tidal acceleration. Since the galaxy rotates, and radial and transverse tidal acceleration cancel, the pressure of a real galaxy is less than that shown in Figure 4(a). Note that when the galaxy disk is perpendicular to the orbit, Figure 3(a) indicates the real tidal acceleration because there is no radial tidal acceleration.

Using the pressure evolution, Equation (1) is solved. The evolution of a model galaxy for $t<0$ is the same as that in $\$ 3.1$. The evolution of the SFRs is shown in Figure 3(b) and 4(b). The SFRs become maximum before the galaxy reaches the center of the cluster. At that time, the galaxy is located at $50 \lesssim r \lesssim 200 \mathrm{kpc}$ (Figure 2 ). Since the time scales of the pressure increase are $\sim 2 \times 10^{8}$ yr and $P$ becomes larger then $100 \mathrm{P}_{\odot}$ for all models, the evolution of the SFRs is similar among them (see $\$ 3.1$ ). Thus, it is common among the models that the maximum SFR is three to four times as much as the initial SFR.

The evolution of $M_{\text {tot }}$ is shown in Figure 3(c) and 4(c). During the passage through the central region of a cluster, $M_{\text {tot }}$ decreases to $\lesssim 1 / 5$ of the initial value.

\subsection{Pressure from the Intracluster Medium}

Numerical simulations of rich cluster evolution show that the local static pressure around a significant fraction of galaxies experienced a rapid increase at high redshift (Evrard 1991). Thus, the change of SFR due to this pressure increase is investigated. The evolution of the pressure is shown in Figure $5(\mathrm{a})$; for $0<t<3 \times 10^{8} \mathrm{yr}, P=10^{2 t / \mathrm{Gyr}} \mathrm{P}_{\odot}$, and for $t>3 \times 10^{8} \mathrm{yr}, P=10^{0.6} \mathrm{P} \odot$. This corresponds to the most rapid increase of pressure in Figure 1 of Evrard (1991). The evolution of a model galaxy for $t<0$ is the same as that in $\$ 3.1$. Figure 5 also shows the evolution of SFR and $M_{\text {tot }}$. The SFR increases by at most $70 \%$. Thus, the increase of static pressure may not induce observable changes in color. 


\subsection{High-Speed Encounters}

Moore et al. (1996) propose that multiple high speed encounters between galaxies (galaxy harassment) change the structure of galaxies in clusters. Therefore, the effect of high-speed encounters on the SFR of a disk galaxy is considered.

In the first place, a single encounter is considered. As an extreme case, a penetrating encounter is calculated. The derivation of $P(t)$ is the same as that in $\S 3.2$ except for the mass distribution of a perturber.

The mass distribution of a perturber is

$$
M_{\text {per }}(<r)=1.0 \times 10^{11} \mathrm{M}_{\odot}\left[\ln \left[\frac{r}{r_{\text {per }}}+\left(1+\frac{r^{2}}{r_{\text {per }}^{2}}\right)\right]^{1 / 2}-\frac{r / r_{\text {per }}}{\left(1+r^{2} / r_{\text {per }}^{2}\right)^{1 / 2}}\right],
$$

for $r<50 \mathrm{kpc}$. The characteristic radius $r_{\text {per }}$ is $5 \mathrm{kpc}$. For $r>50 \mathrm{kpc}$, $M_{\text {per }}(<r)=2.0 \times 10^{11} \mathrm{M}_{\odot}$. The evolution of a model galaxy for $t<0$ is also the same as that in 3.1 . At $t=0$, a model disk galaxy is located at $r=100 \mathrm{kpc}$, and the relative velocity to the perturber is $1000 \mathrm{~km} \mathrm{~s}^{-1}$. Because of the symmetry between the approach and recession phases of the model galaxy, only the transverse tidal acceleration is calculated. The results are shown in Figure 5. The model galaxy passes the center of the perturber at $t \sim 10^{8} \mathrm{yr}$. Since the kinetic pressure does not change significantly, the SFR rises by only $13 \%$.

In this argument, however, the ISM of the perturber is not taken into account. If it has its own ISM with density $\rho_{p}$, the ram pressure $\rho_{p} V^{2}$ acts on molecular clouds in the model galaxy. For example, when $\rho_{d}=1.67 \times 10^{-24} \mathrm{~g} \mathrm{~cm}^{-3}$ for $r<5 \mathrm{kpc}$ and $\rho_{d}=0$ for $r>5$ $\mathrm{kpc}$, the SFR temporarily increases more than ten times larger than the initial value. The formation of a ring or tidal arms due to a close encounter will also affect the SFR. However, Moore et al. (1996) indicate that such close encounters $(<30 \mathrm{kpc})$ are extremely rare in a cluster. Thus, a single high speed encounter does not drive the BOE.

On the other hand, galaxy harassment would induce star formation activity in many galaxies in a cluster. Using the results of Moore, Lake, and Katz (1998) and Lake, Katz, \& Moore (1998), the change of SFR due to the harassment is calculated.

Galaxy harassment has a great influence on galaxies smaller than the Galaxy. Thus, the initial conditions of a model galaxy are different from those considered above. At $t=0$, the SFR is $1.2 \mathrm{M}_{\odot} \mathrm{yr}^{-1}$ and $M_{\text {tot }}=3.7 \times 10^{9} \mathrm{M}_{\odot}$, which correspond to those of the model galaxy in Moore et al. (1998) and Lake et al. (1998). The initial value of pressure is taken to be $P=0.1 \mathrm{P}_{\odot}$ in order to balance the cloud destruction rate with the formation rate. Lake et al. (1998) show that most of the gas of the harassed galaxy is driven to the region 
within $1 \mathrm{kpc}$ of the center within $3 \mathrm{Gyr}$. In the extreme case, half of the mass is transfered in an interval of 100 - 200 Myr. Assuming that $60 \%$ of the gas is driven to the region within $1 \mathrm{kpc}$ of the center and that the hight of the gas disk is $200 \mathrm{kpc}$, the gas density is $2.4 \times 10^{-22} \mathrm{~g} \mathrm{~cm}^{-3}$. Since the effective velocity is $\sim 130 \mathrm{~km} \mathrm{~s}^{-1}$ (Fig. 2 in Moore at al. [1998]), the pressure of the central region of the harassed galaxy is $1.0 \times 10^{4} \mathrm{P} \odot$. Thus, the evolution of the model galaxy is assumed to be $P(t)=10^{10(t / \mathrm{Gyr})-1} \mathrm{P}_{\odot}$ (Figure 5a). Using this and Equation (11), the evolution of the model galaxy can be calculated. The results are shown in Figure 5(b) and 5(c). The history of SFR shows that galaxy harassment can induce strong star formation activity.

\section{Conclusions}

In this paper, the influence of environmental effects on the star formation rate (SFR) of disk galaxies in clusters was quantitatively estimated. The constructed model treats the response of star formation to local pressure variance. Since it does not care about the origin of pressure, it can be used to investigate various candidates for the Butcher-Oemler effect.

General analysis indicates that the SFR increases significantly when the pressure of molecular clouds rises above $\sim 10 \mathrm{P}_{\odot}$ in $\sim 10^{8}$ yr but reaches the ceiling at $\sim 100 \mathrm{P}_{\odot}$. This is because the destruction time of most molecular clouds in the galaxy becomes $\lesssim 10^{8}$ yr.

In the analysis of a disk galaxy tidally affected by the cluster, it is found that the maximum SFR is around three or four times as much as that of a field galaxy, and does not depend greatly on the mass profiles of the cluster and the type of the tidal force. The SFR becomes maximum before the galaxy reaches the center of the cluster. At that time, the distance from the center is $50 \lesssim r \lesssim 200$. Fritze $-\mathrm{v}$ Alvensleben \& Gerhard (1994) have investigated the color evolution during and after starbursts for many types of galaxy. Their results show that the color of a galaxy significantly becomes bluer for $\sim 10^{8}$ yr after the burst if the gas mass fraction just before the burst is $\sim 10 \%$, the duration of the burst is $\sim 10^{8} \mathrm{yr}$, and most of the gas is consumed during the burst. Therefore, if the tidally affected galaxies are responsible for the Butcher-Oemler effect (BOE), the blue galaxies should be found $\lesssim 300 \mathrm{kpc}$ from the center of the cluster (Figure 2). It is confirmed that even if the initial velocity of the model galaxy is $2000 \mathrm{~km} \mathrm{~s}^{-1}$, which is larger than the virial velocity of the model cluster, this prediction does not significantly change. However, recent observations are inconsistent with this prediction. In A2317, the blue population decreases remarkably inside $300 \mathrm{kpc}$ (Rakos, Odell, \& Schombert 1997). Since this trend is observed in other distant clusters (Abraham et al. 1996 ; Balogh et al. 1997), it appears that tidal 
force from the potential well of a cluster does not induce the BOE.

As a galaxy plows into the hot intracluster medium, the external pressure rapidly rises. However, the rise of local pressure based on numerical calculation done by Evrard (1991) does not appear to induce starbursts because the maximum of the pressure is small. This is consistent with the recent observation of a merging cluster (Tomita et al. 1996).

The velocity perturbation induced by a single high-speed encounter is too small to affect the SFR of a disk galaxy. However, successive high-speed encounters between galaxies (galaxy harassment) lead to gas inflow and strong star formation activity. Thus, galaxy harassment may be a likely explanation for the star-forming galaxies observed in high-redshift clusters (Oemler, Dressler, \& Butcher 1997 ; Couch et al. 1998 ; Smail et al. 1998). In order to know whether galaxy harassment is the main origin of BOE, it is required to estimate the fraction of galaxies in which the gas accumulates in the center. The spatial distribution of blue galaxies predicted by the galaxy harassment model may be consistent with the observations, because galaxies in the core of clusters will be older than galaxies at the edges.

On the other hand, the BOE may not be excess star formation induced by the infall process or internal tides in clusters of galaxies. Abraham et al. (1996) suggest that the $\mathrm{BOE}$ is due to the increased rate of infall of bluer field galaxies at higher redshift, and that the star formation is truncated without an increase. A model including ram-pressure stripping and evolution of intracluster medium will be needed to investigate this idea.

I would like to thank S. Sasaki and T. Tsuchiya for their helpful comments. I wish to thank S. Inoue for his assistance. I am also grateful to an anonymous referee for several suggestions that improved this paper. This work was supported in part by the JSPS Research Fellowship for Young Scientists.

\section{REFERENCES}

Abraham, R. G., et al. 1996, ApJ, 471, 694

Bahcall, N. A. 1981, ApJ, 247, 787

Balogh, M. L., Morris, S. L., Yee, H. K. C., Carlberg, R. G., \& Ellingson, E. 1997, ApJ, 488, L75

Butcher, H., \& Oemler, A., Jr. 1978, ApJ, 219, 18

Byrd, G., \& Valtonen, M. 1990, ApJ, 350, 89 
Couch, W. J., Barger, A. J., Smail, I., Ellis, R. S., \& Sharples, R. M. 1998, ApJ. 497, 188

Dressler, A. \& Gunn, J. E. 1983, ApJ, 270, 7

Elmegreen, B. G. 1989, ApJ, 338, 178

Elmegreen, B. G., \& Efremov, Y. N. 1997, ApJ, 480, 235

Evrard, A. E. 1991, MNRAS, 248, 8p

Fritze -v Alvensleben. U., \& Gerhard, O. E. 1994, A\&A, 285, 751

Güsten, R, \& Mezger, P. G. 1983, Vistas in Astron., 26, 159

Harris, W. E., \& Pudritz, R. E. 1994, ApJ, 429, 177

Henriksen, M. J., \& Byrd, G. 1996, ApJ, 459, 82

Lake, G., Katz, N., \& Moore, B. 1998, ApJ, 495, 152

Makino, N., Sasaki, S, \& Suto, Y. 1998, ApJ, in press

Maeder, A. 1994, A\&A, 264, 105

Mihara, K., \& Takahara, F. 1994, PASJ, 46, 447

Moore, B., Katz, N., Lake, G., Dressler, A., \& Oemler A. 1994, Nature, 379, 613

Moore, B., Lake, G., Katz, N. 1998, ApJ, 495, 139

Navarro, J. F., Frenk, C. S., \& White, S. D. M. 1995, MNRAS, 275, 720

Navarro, J. F., Frenk, C. S., \& White, S. D. M. 1996, ApJ, 462, 563

Oemler, A., Dressler, A., \& Butcher, H. R. 1997, ApJ, 474, 561

Rakos, K. D., Odell, A. P., \& Schombert, J. M. 1997, ApJ, 490, 194

Smail, I., Edge, A. C., Ellis, R. S., \& Blandford, R. D. 1998, MNRAS, 293, 124

Larson, R. B. 1987, In Starbursts and Galaxy Evolution, ed. Trinh Xuan Thuan, T. Montmerle, \& J. Tran Thanh Van (France: Editions Frontieres)

Tomita, A., Nakamura, F. E., Tanaka, T., Nakanishi, K., Takeuchi, T., Ohta, K., and Yamada, T. 1996, AJ, 111, 42

Valluri, M. 1993, ApJ, 408, 57 


\section{Figure Captions}

Fig.1 - Histories of (a) pressure (b) star formation rate, and (c) total mass of molecular clouds.

Fig.2 - Orbits of radially infalling galaxies in the three potential wells. Solid line; lensing potential (GL), dotted line; King model (King), dashed line; the one derived by Navarro et al.(NFW).

Fig.3 - Histories of (a) pressure (b) star formation rate, and (c) total mass of molecular clouds for a galaxy affected by the transverse tidal acceleration. Solid line; lensing potential (GL), dotted line; King model (King), dashed line; the one derived by Navarro et al.(NFW).

Fig.4 - Same as in Fig.3 but for radial tidal acceleration.

Fig.5 - Histories of (a) pressure (b) star formation rate, and (c) total mass of molecular clouds for a galaxy affected by the pressure of intracluster medium (ICM; solid line), a single high-speed encounter (HE; dotted line), and galaxy harassment (GH; dashed line). 


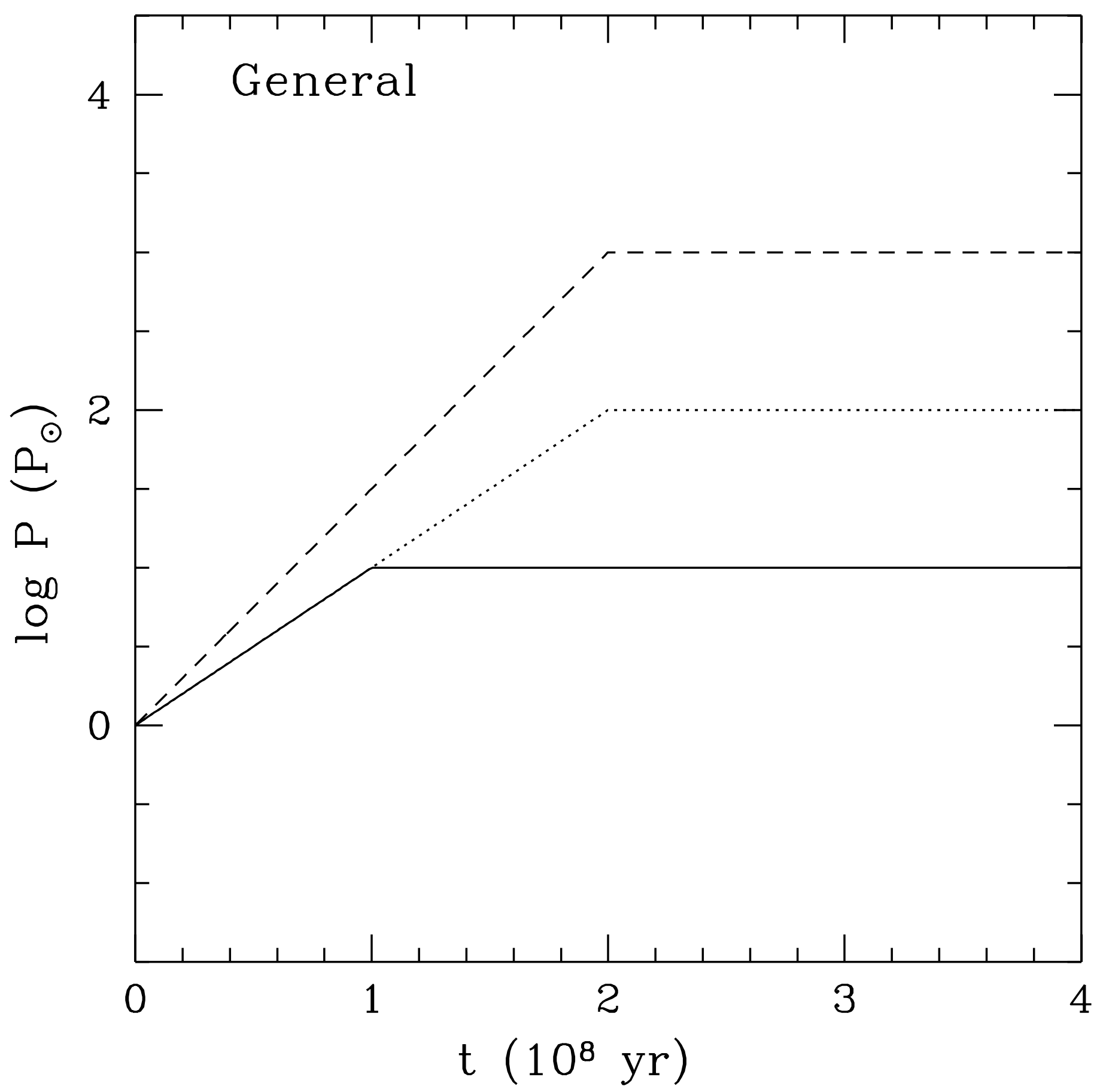




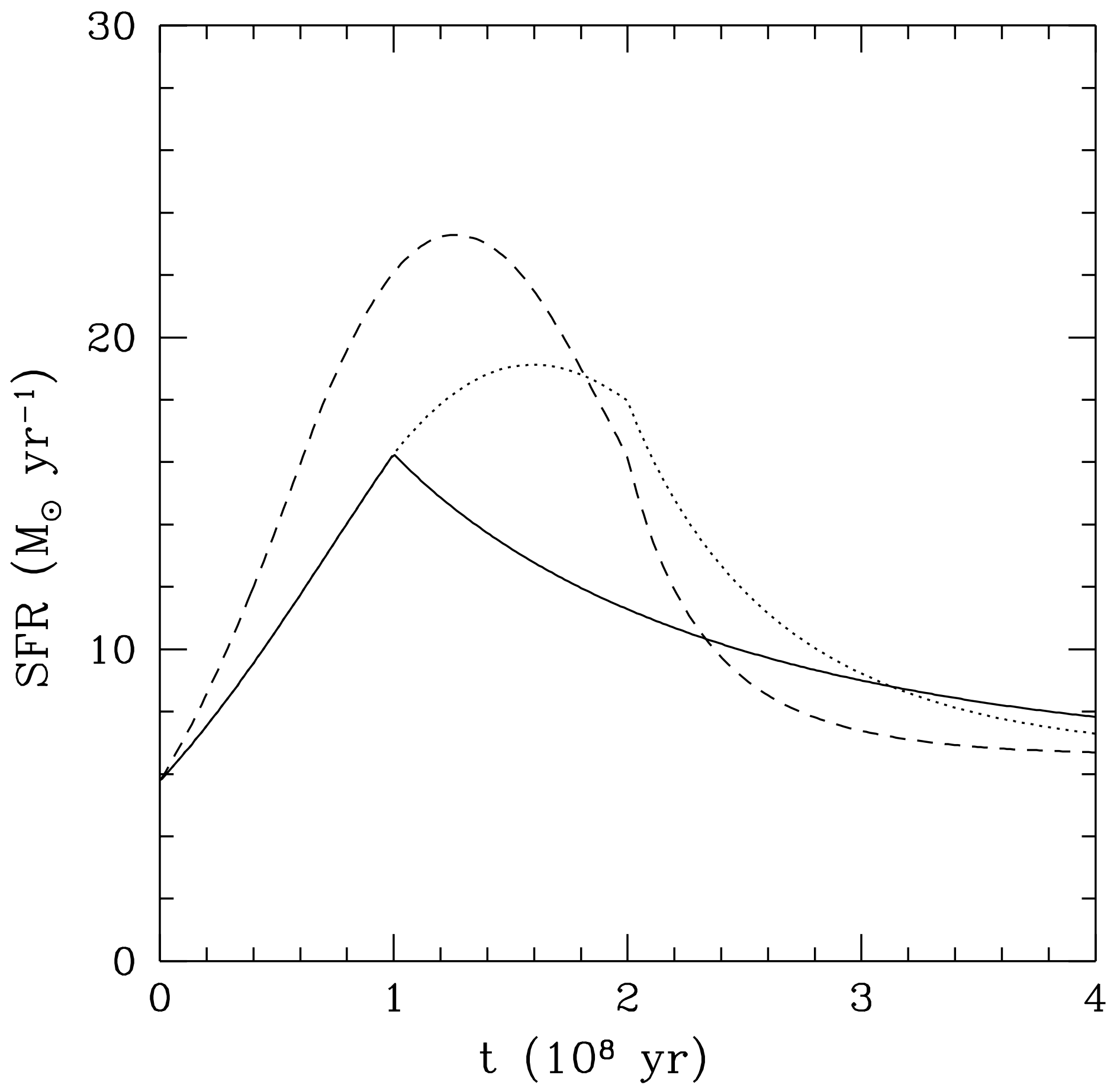




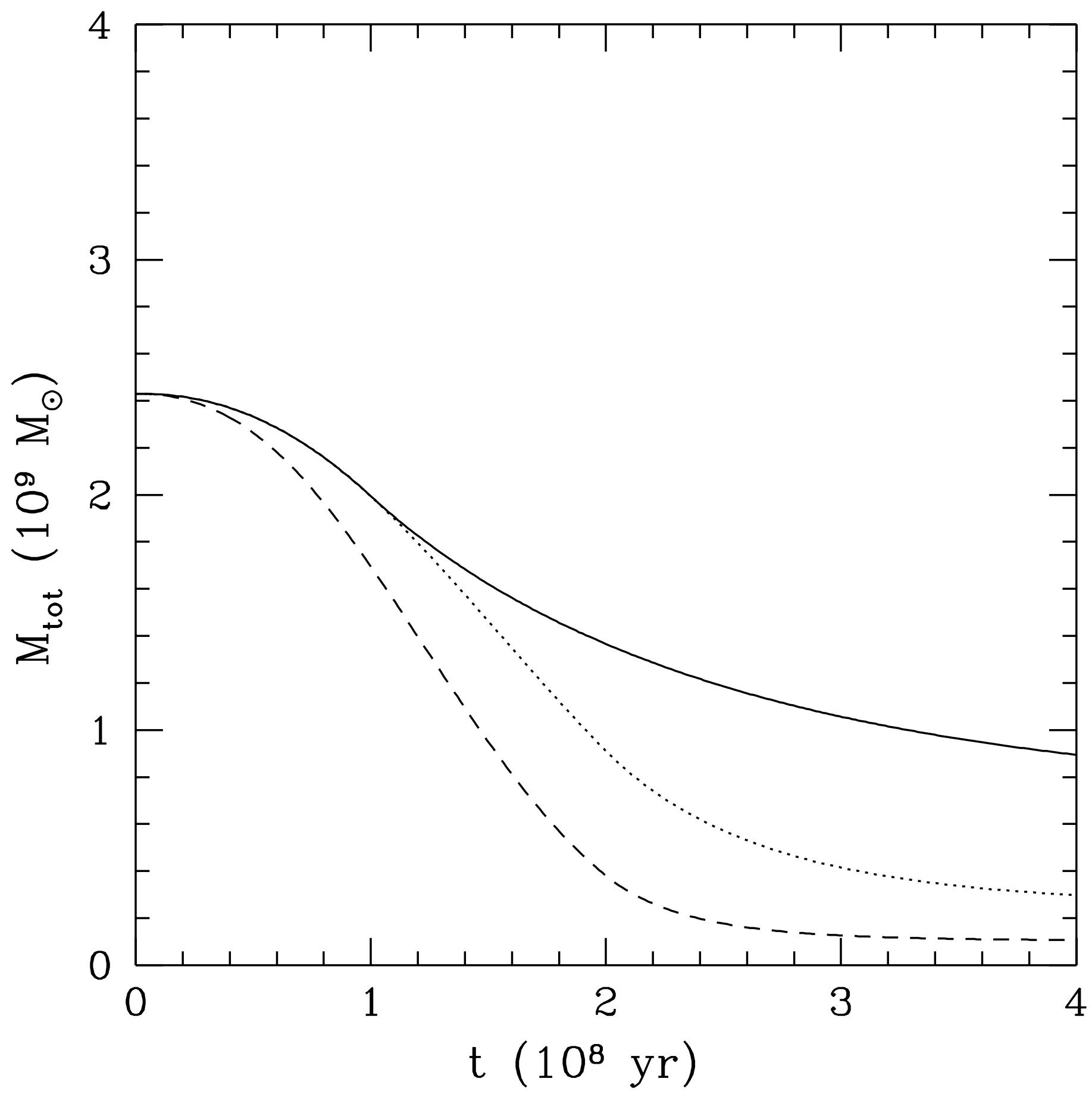




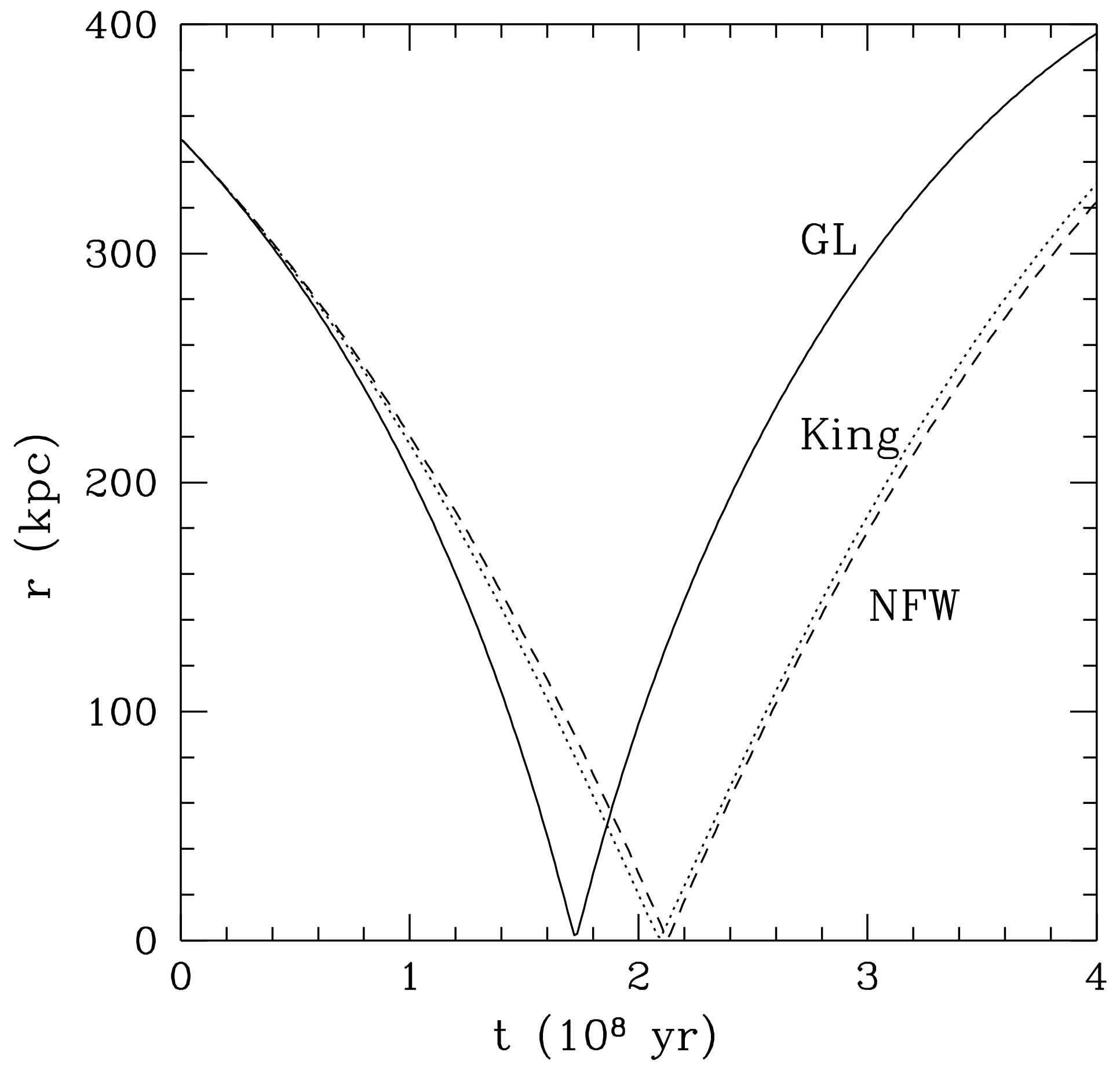




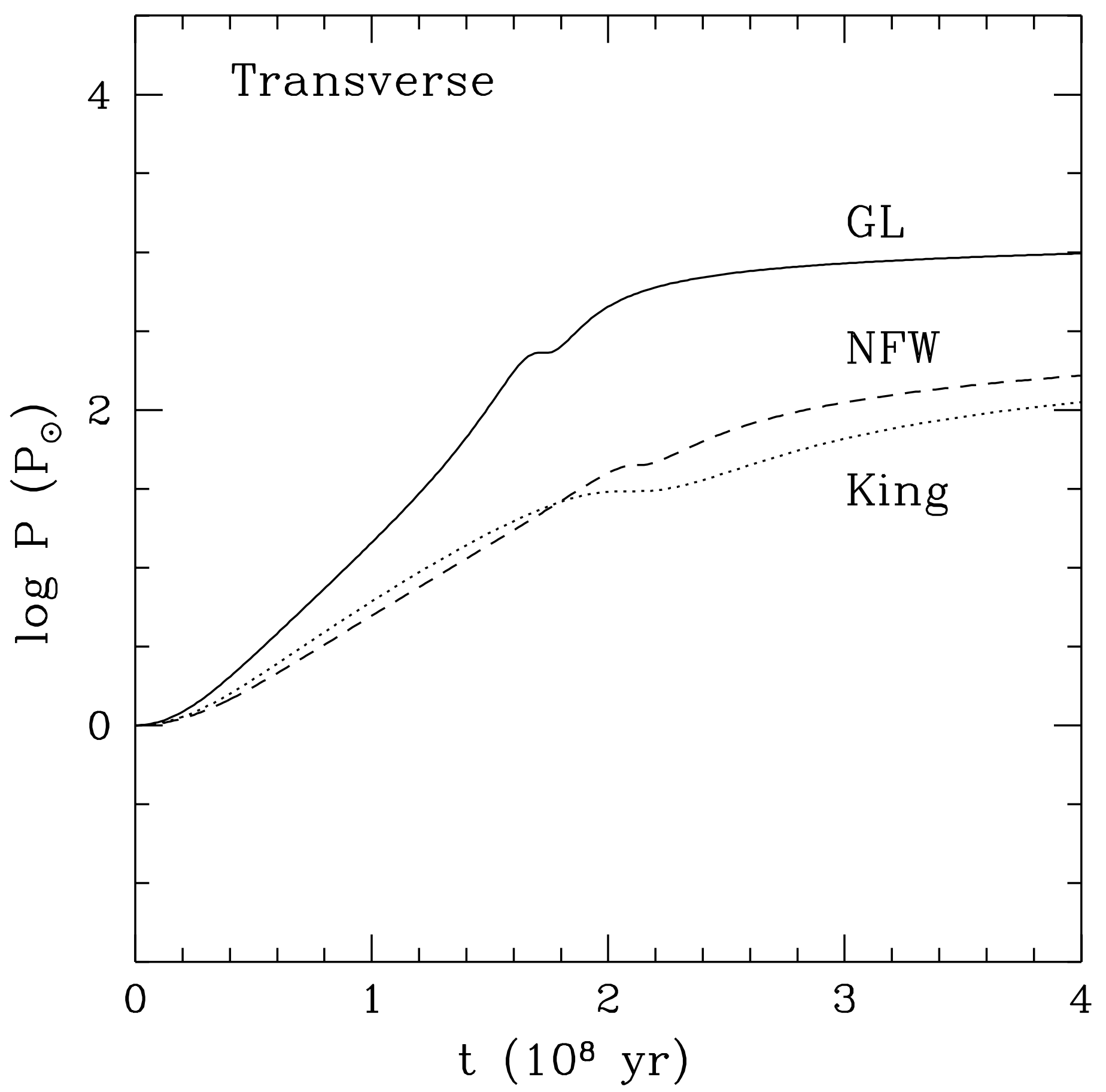




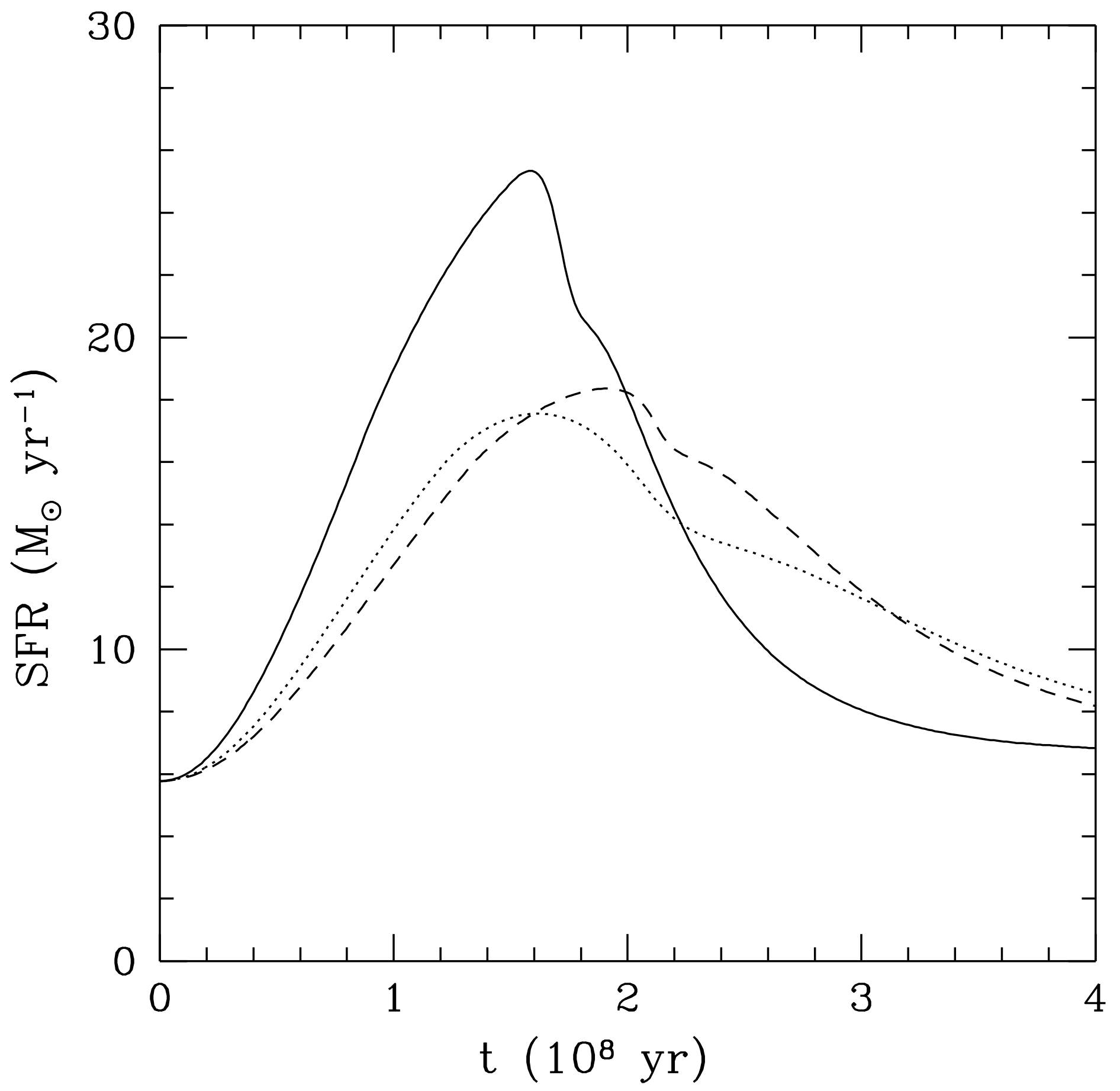




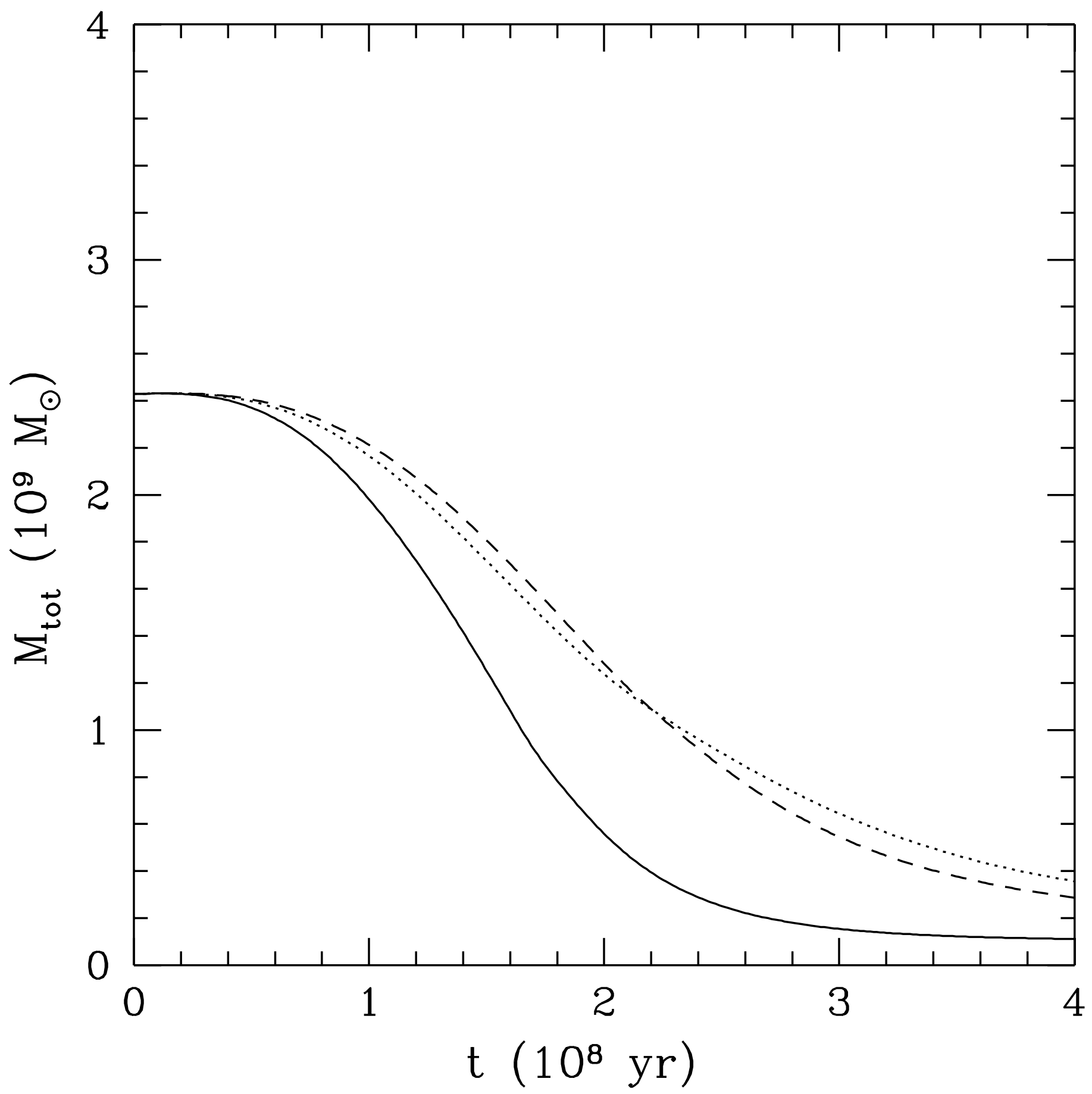




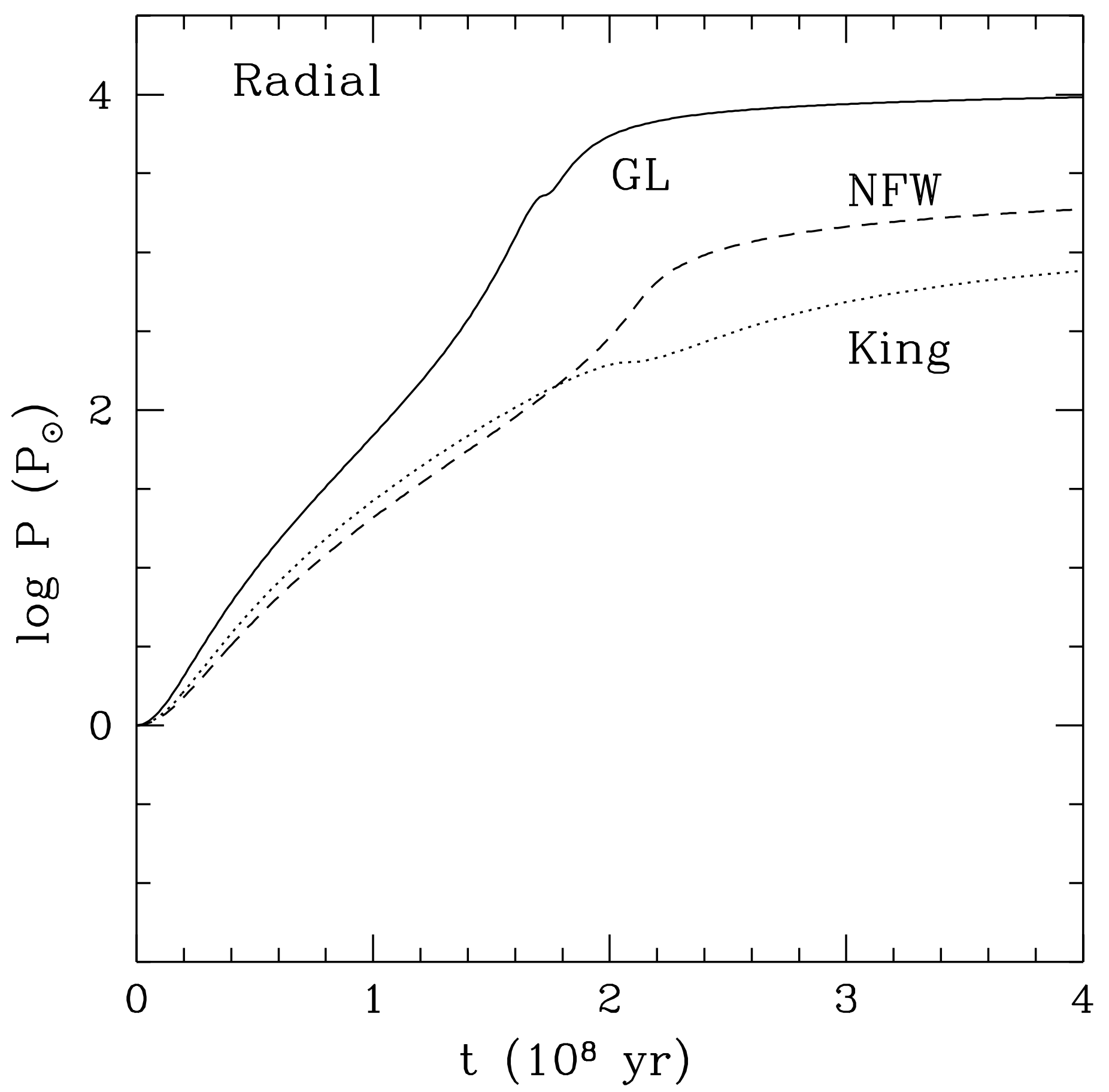




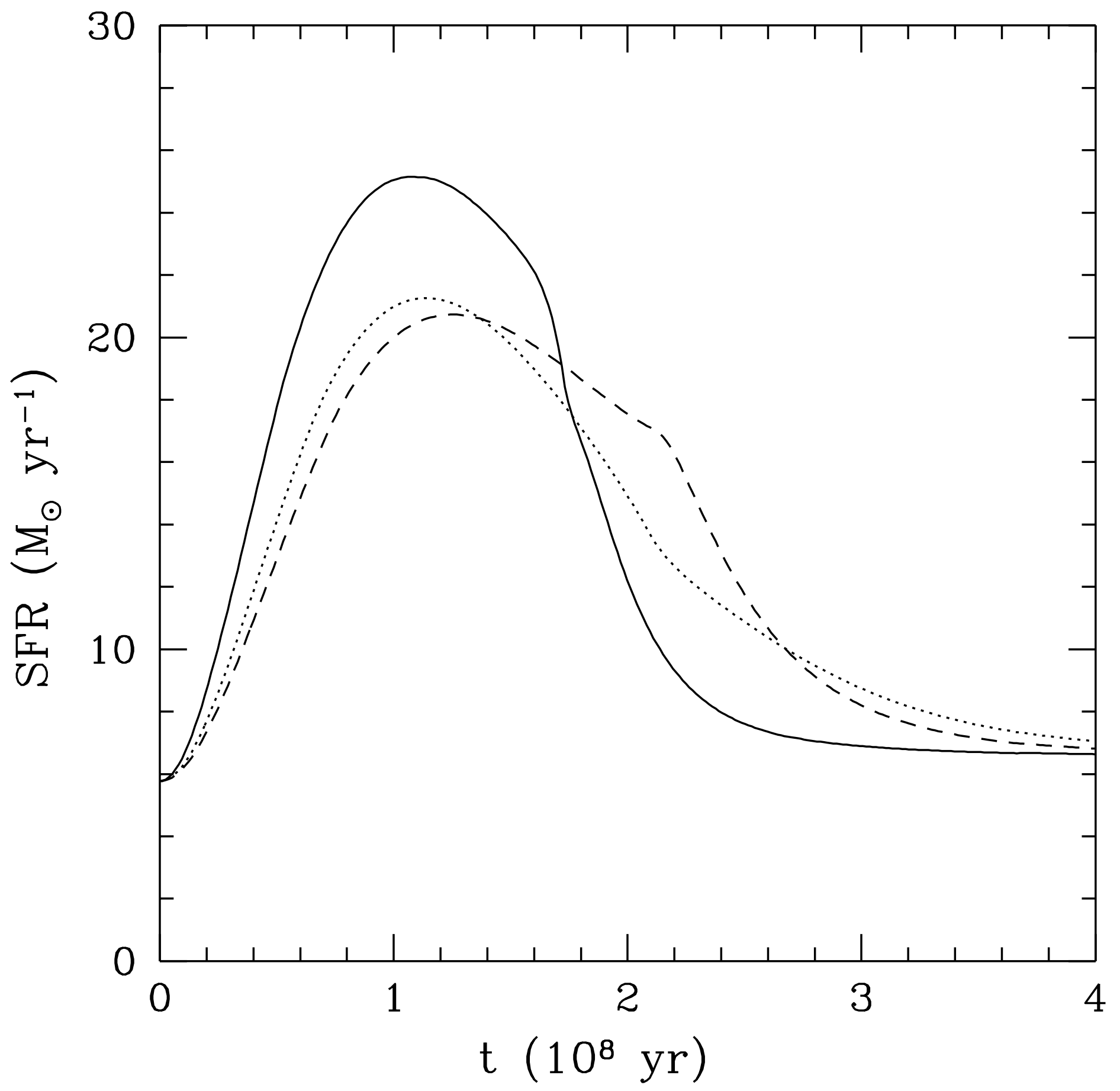




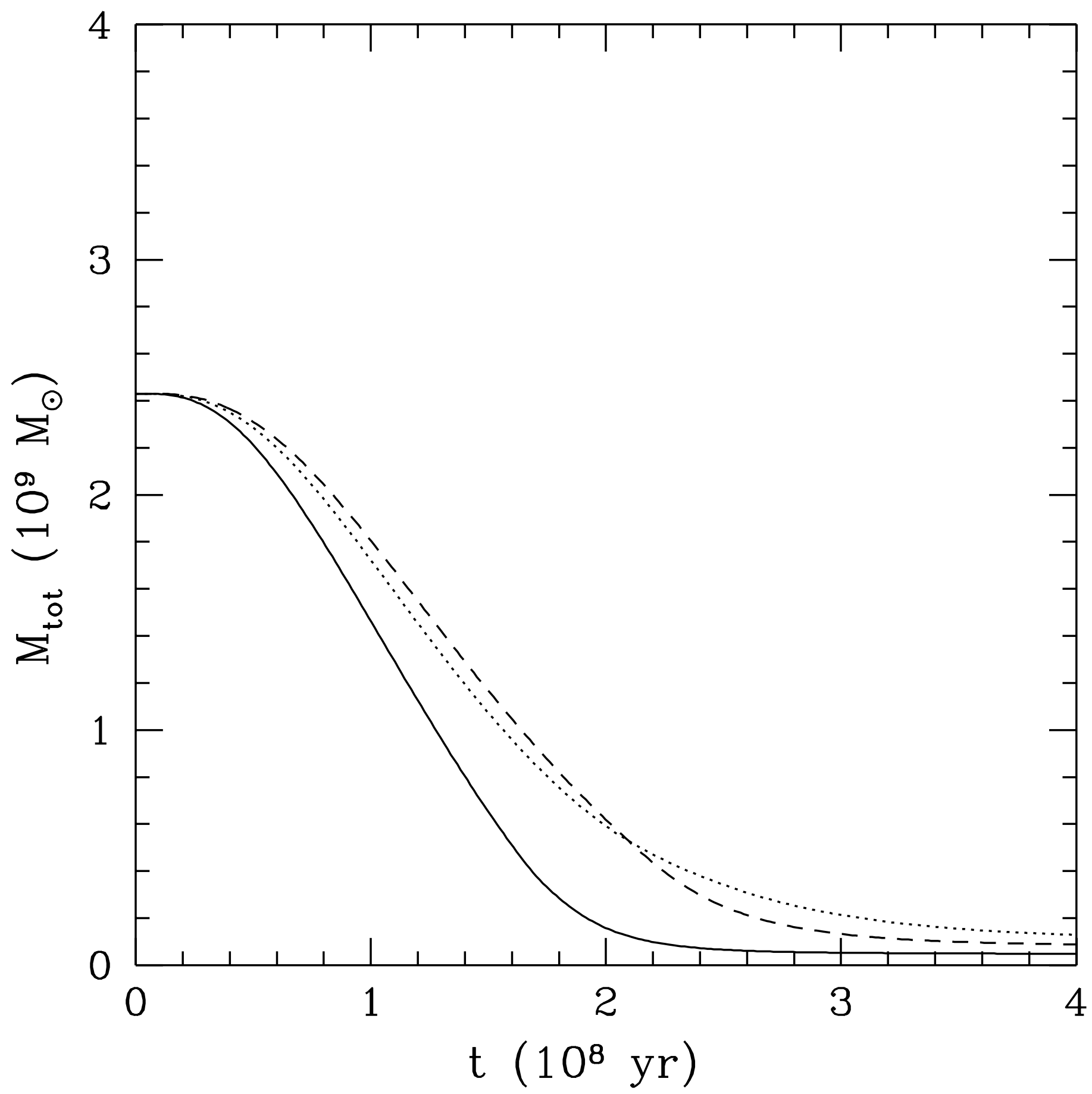




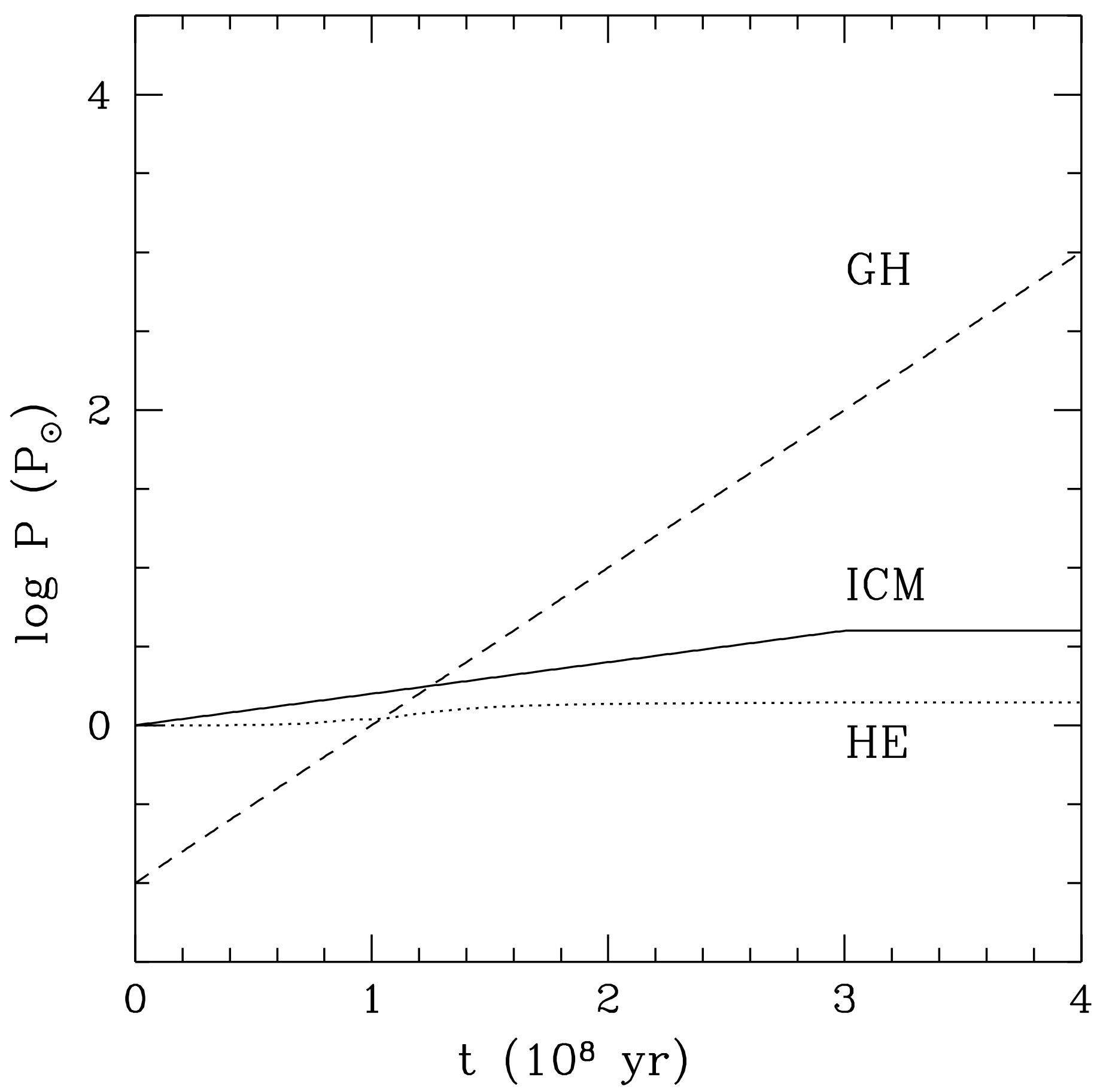




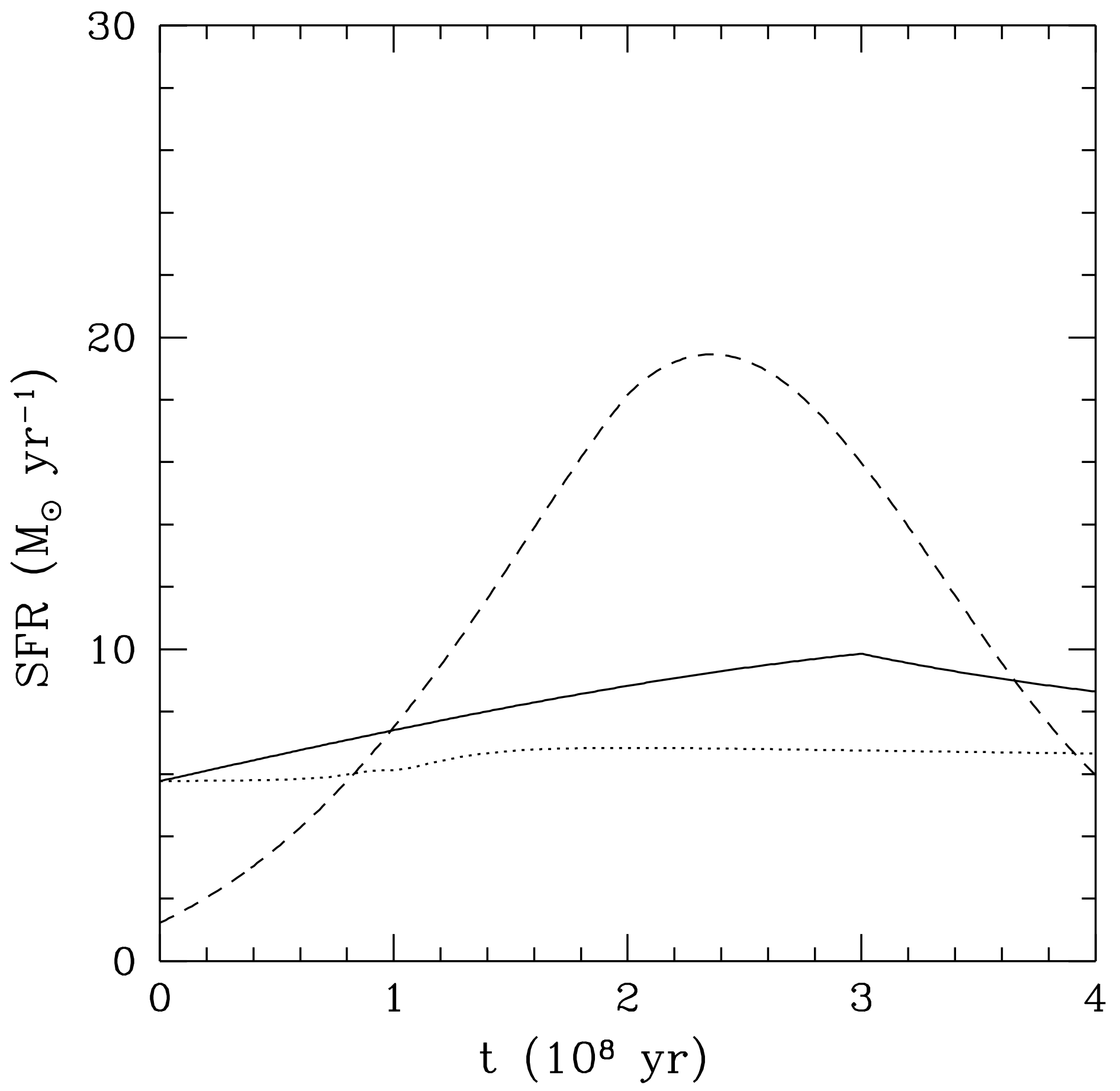




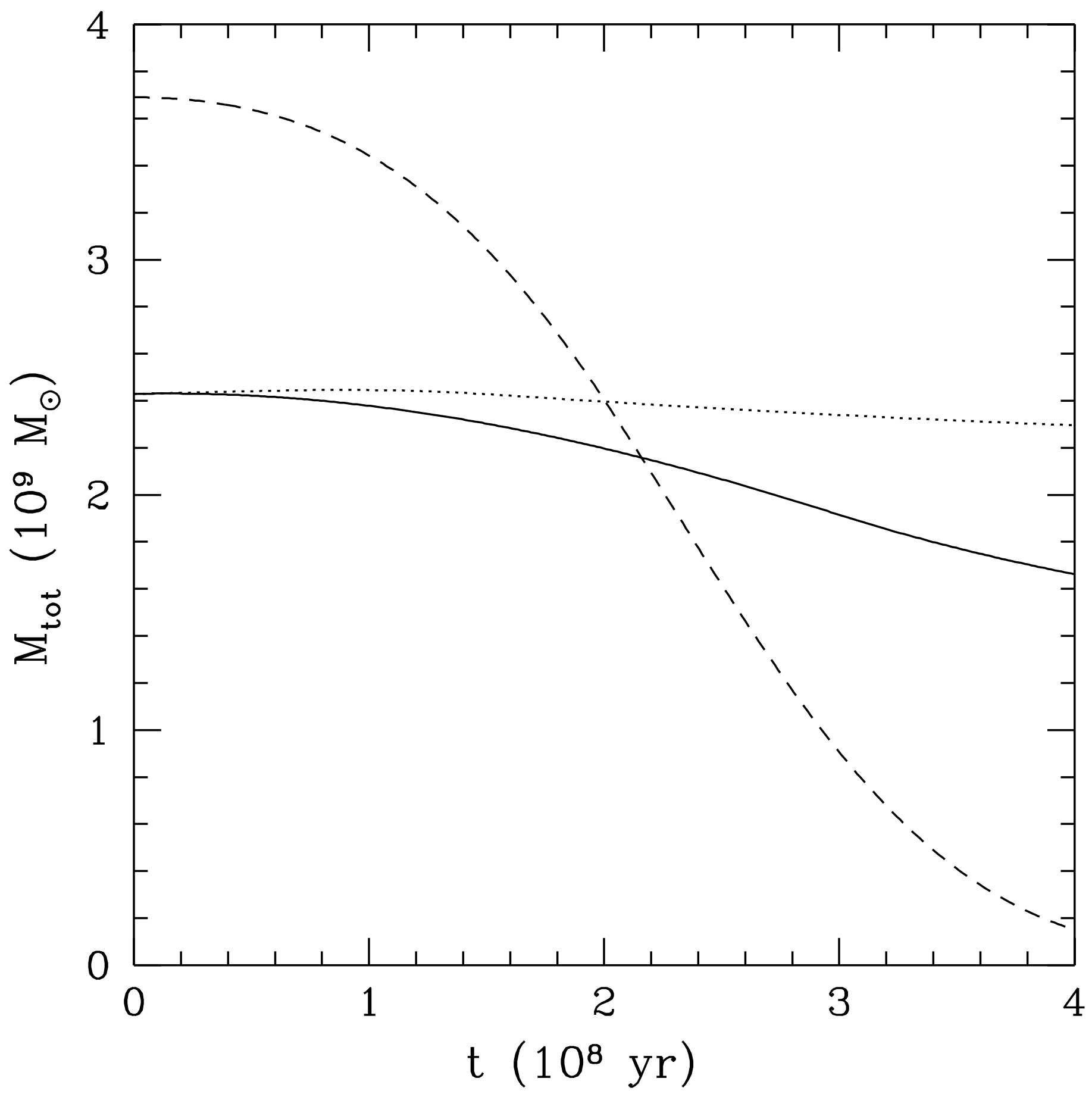

